\title{
EL PRODUCTO BRUTO INTERNO (PBI) Y SU RELACIÓN CON LOS INGRESOS TRIBUTARIOS EN EL PERÚ 2007-2018
}

\author{
THE INTERNAL GROSS PRODUCT (GDP) AND ITS RELATIONSHIP WITH TAX \\ INCOME IN PERU 2007-2018
}

\author{
José Rojas Fernández \\ Universidad Nacional Federico Villarreal \\ Lima, Perú \\ ORCID: http://orcid.org/0000-0002-0695-2526 \\ Correo electrónico: Joserojas44148@hotmail.com
}

\author{
Luis Arturo Vizcarra Domínguez \\ Universidad Nacional Mayor de San Marcos \\ Lima, Perú \\ ORCID: http://orcid.org/0000-0002-9976-4400
}

\section{RESUMEN}

Objetivo: Analizar la relación entre el Producto Bruto Interno y los Ingresos Tributarios que recauda el Gobierno Central. Método: El enfoque de la investigación es cuantitativo de tipo descriptivo y correlacional, cuyo diseño es no experimental y longitudinal, es decir, se trabaja con información documental de la recaudación tributaria y del producto interno desde el año 2007 hasta el 2018 de la Superintendencia Nacional de Aduanas y de Administración Tributaria (SUNAT). Resultados: No hay variabilidad en el producto bruto interno como tampoco en la recaudación tributaria; los datos son homogéneos en cada variable. Conclusiones: $\mathrm{Se}$ concluye que sí existe una relación significativa entre el producto bruto interno y los Ingresos Tributarios, este resultado se confirmó con el coeficiente de correlación de Pearson, el cual fue 0,9401, definida como alta correlación entre las dos variables indicadas.

Palabras clave: Producto; ingresos; tributos; demanda interna; inversión pública

\begin{abstract}
Objective: To analyze the relationship between the Gross Domestic Product and the Tax Revenue collected by the Peruvian State. Method: The research approach is quantitative descriptive and correlational type, whose design was non-experimental and longitudinal, that is, documentary information was collected on tax collection and the internal product from 2007 to 2018 of the National Superintendence Customs and Tax Administration (SUNAT). Results: There is no variability in the gross domestic product as well as in the tax collection; The data is homogeneous in each variable. Conclusions: It is concluded that there is a significant relationship between the gross domestic product and the Tax Revenue, this result was confirmed with the Pearson correlation coefficient, which was 0.9401 , defined as a high correlation between the two indicated variables.
\end{abstract}

Keywords: Product; income; taxes; domestic demand; public investment. 


\section{INTRODUCCIÓN}

En el Perú, la recaudación tributaria comprende el monto de los impuestos pagados al Gobierno Central menos las devoluciones que se realiza a los contribuyentes por este mismo concepto, y se mide a través de los Ingresos Tributarios del Gobierno Central (ITGC).

Los principales impuestos que conforman la recaudación tributaria según Lahura y Castillo (2018) son:

El impuesto a los ingresos (el impuesto a la renta pagado por las personas jurídicas y personas naturales), el impuesto general a las ventas (IGV), el impuesto selectivo al consumo (ISC), el impuesto a las importaciones (aranceles de aduanas y sobretasa arancelaria) y otros ingresos tributarios (p. 34).

Moreno (2018) refiere que:

Para todas las economías, la recaudación tributaria es uno de los temas de mayor importancia y es que a través de ella, el Estado puede asumir sus obligaciones y crear las condiciones necesarias para el desarrollo de un país, mejorando la calidad de vida de los ciudadanos. También es importante que se establezcan impuestos justos sin que esta afecte el dinamismo de la economía (productividad) y que los ciudadanos puedan tomar un mayor control de su dinero (p.11).

Además, Ordoñez (2014) afirma que:

El sustento del Presupuesto Público son sus ingresos por diferentes fuentes de financiamiento, pero especialmente los ingresos tributarios. En la medida que crezca la actividad económica del país, se espera que exista una mayor recaudación tributaria, tanto directa como indirecta. Por eso nos parece importante, entonces, determinar de qué manera el crecimiento económico del país incide en una mayor recaudación tributaria que permita al Estado cumplir las innumerables funciones y tareas que tiene asignadas (p.1)

En el 2017, el crecimiento promedio del PBI del Perú fue $3,3 \%$ menor que en años anteriores, esto se debió a que la evasión y elusión tributaria de una economía informal originan una menor recaudación de Ingresos Tributarios y una disminución de la tasa de crecimiento del PBI. La recuperación de las tasas de dicho crecimiento es entendida como reactivación de la economía.

Parodi (2019) explica que, para reactivar la economía, se debe impulsar cuatro componentes, a saber: el consumo privado, el gasto público (compuesto por el consumo público más inversión pública), la inversión privada y las exportaciones. Si estas aumentan, sobre todo el consumo privado, la economía se ve favorecida.

En la Memoria 2018 publicada por el Banco Central de Reserva del Perú, se señala que el consumo privado representó el 66,35\% de la demanda interna. En este escenario, las personas que desean consumir en mayor medida necesitan de más dinero, el cual se obtiene a partir de más empleos o mayores sueldos por parte de las empresas de todo tamaño; con ese mayor dinero que se gasta, se reactiva el consumo privado y, así, la producción.

Por parte de la inversión privada, esta es realizada tanto por la gran minería como el pequeño comerciante y, junto con la inversión pública, tienen que mostrar aumentos sostenidos en el tiempo.

Los factores que aumentan la inversión son internos (por ejemplo, la incertidumbre política del país) y externos (la guerra comercial entre los Estados Unidos y China, la desaceleración europea, las tensiones ante la salida del Reino Unido de la Unión Europea, etc.). Al ser difícil tomar decisiones en esos contextos, los agentes económicos postergan las decisiones ante el hecho de no tener idea sobre lo que pasará (Parodi, 2019).

En la investigación se ha formulado el siguiente problema: ¿cuál es la relación entre los Ingresos Tributarios que recauda el Gobierno Central y con el Producto Bruto Interno?

Para proceder a responder, primero se debe comprender qué representa el PBI, para lo cual el Banco Central de Reserva del Perú lo define como «el valor total de la producción corriente de bienes y servicios finales dentro de un país durante un periodo de tiempo determinado. Incluye por lo tanto la producción generada por los nacionales y los extranjeros residentes en el país» (Banco Central de Reserva del Perú, 2019).

El PBI representa el valor de la producción final de bienes y servicios en un período. Según De Gregorio (2007):

La idea de medir la producción final es que queremos evitar contar los bienes intermedios; es decir, aquellos que se usan en la producción de otros bienes. De esta forma podremos evitar la doble, o más bien múltiple, contabilidad de bienes (p.14).

Por lo tanto, reactivar la economía es importante por dos razones, primero, porque genera empleo, ya que, si se produce más, se espera que las empresas demanden más personas y, para que esto ocurra, deben tener calificaciones e insertarse así al mercado natural. En segundo lugar, producir más conlleva aumentos en la recaudación tributaria, por lo que se aumenta la capacidad del gasto del Gobierno. 


\section{MATERIALES Y MÉTODO}

El enfoque de la investigación es cuantitativo, es decir, es un estudio objetivo y sistemático. También se realizaron pruebas de hipótesis correlacionales para demostrar los supuestos planteados al inicio del estudio. El tipo de investigación es descriptivo porque se realizó un análisis exploratorio de los datos y es correlacional porque se relacionaron las variables: el Producto Bruto Interno y los Ingresos Tributarios que recauda el Gobierno Central.

El diseño de la investigación es no experimental de tipo longitudinal, ya que se recolectó información documental de la Superintendencia Nacional de Aduanas y de Administración Tributaria (SUNAT) acerca del producto bruto interno y los Ingresos Tributarios que recauda el Gobierno Central desde el 2007 hasta el 2018.

La fuente de información es secundaria, ya que los datos fueron de documentos recolectados a través del tiempo, es decir, el PBI y los Ingresos Tributarios que recauda el Gobierno se obtuvieron de la SUNAT desde el 2007 hasta el 2018.

\section{RESULTADOS}

A continuación, se presenta el análisis exploratorio de datos con el uso de las medidas estadísticas en la Tabla 1.

Se muestra que el promedio del PBI y de los Ingresos Tributarios que recauda el Gobierno es de 522 271,33 y 78 946,26 (en millones de soles), respectivamente. También se definió la desviación estándar y el grado de dispersión del PBI respecto a su promedio que es de 139 420,61 (en millones de soles), por lo cual, la variabilidad de los Ingresos Tributarios que recauda el Gobierno Central respecto a su promedio es 17789.95 (en millones de soles). La mediana del PBI es 527 420,5 (en millones de soles), valores menores a la mediana que representa el 50\% de los datos y valores mayores representa el otro $50 \%$ de los datos, por ello se deduce que el PBI es asimétrica positiva porque hay mayor variabilidad de los datos en los valores superiores a la mediana; esto se confirma teóricamente obteniendo la asimetría positiva del PBI, esto es 0,049, cuyo valor es mayor que cero. La mediana de los Ingresos Tributarios es de 86764,35 (en millones de soles), por lo cual se deduce que tiene una asimetría negativa porque hay mayor variabilidad de los datos en los valores inferiores a la mediana, lo cual se confirma teóricamente una asimetría negativa en $-0,425$, cuyo valor es menor que cero.

En los gráficos de cajas, se observa la asimetría del PBI y de los Ingresos Tributarios, como también si hay datos atípicos o extremos.

En la Figura 1, se observa que el PBI tiene una asimetría positiva porque los datos están moderadamente sesgados a partir del promedio hacia valores grandes. No hay valores atípicos, es decir, no hay valores que están fuera de la caja. Debido a lo observado en la caja se deduce que no hay variabilidad de los datos del PBI.

En la Figura 2, se muestra que los Ingresos Tributarios que recauda el Gobierno Central tienen una asimetría negativa los datos están moderadamente sesgados a partir del promedio hacia valores pequeños. No hay valores atípicos, todos los datos de los Ingresos Tributarios que recauda el Gobierno están concentrados en la caja. Se deduce que no hay variabilidad de los datos de los Ingresos Tributarios que recauda el Gobierno.

Para demostrar la hipótesis planteada si existe relación entre Producto Bruto Interno y los Ingresos Tributarios que recauda el Estado peruano, se tiene que seguir un método correlacional, el cual es escogido mediante una

Tabla 1.

Medidas Estadísticas del Producto Bruto Interno y el Impuesto Tributario peruano 2007-2018.

\begin{tabular}{|c|c|c|c|}
\hline \multicolumn{2}{|c|}{ Medidas Estadísticas } & Producto Bruto Interno & Impuesto Tributario \\
\hline \multirow{2}{*}{ N } & Válido & 12 & 12 \\
\hline & Perdidos & 0 & 0 \\
\hline \multicolumn{2}{|c|}{ Media } & $522,271.3333$ & $78,946.2583$ \\
\hline \multicolumn{2}{|c|}{ Mediana } & $527,420.5000$ & $86,764.3500$ \\
\hline \multicolumn{2}{|c|}{ Desviación Estándar } & $139,420.61272$ & $17,789.95334$ \\
\hline \multicolumn{2}{|c|}{ Asimetría } & 0,049 & $-0,425$ \\
\hline \multicolumn{2}{|c|}{ Rango } & $421,113.00$ & $52,208.50$ \\
\hline \multicolumn{2}{|c|}{ Mínimo } & $319,693.00$ & $52,381.00$ \\
\hline \multicolumn{2}{|c|}{ Máximo } & $740,806.00$ & $104,589.50$ \\
\hline
\end{tabular}

Fuente: Elaboración propia. 
prueba de normalidad del PBI y los Ingresos Tributarios.

El gráfico Q-Q, llamado también gráfico de cuantiles, brinda una noción visual de la normalidad del PBI y de los Ingresos Tributarios que recauda el Gobierno. En cada gráfico, el eje $\mathrm{X}$ está conformado por los valores observados de cada variable y el eje Y está conformado por la construcción a través de los cuantiles de la distribución normal estándar.

En las Figuras 3 y 4, se observa que los puntos se acercan mucho a la línea, ese detalle da la información que el PBI y los Ingresos Tributarios que recauda el Gobierno se aproximan a una distribución normal.

A continuación, se realiza la prueba de normalidad para el PBI y los Ingresos Tributarios que recauda el Gobierno Central con el fin de probar mediante un análisis teórico si los datos se distribuyen normalmente. Utilizamos la prueba Shapiro-Wilk porque el tamaño de muestra es de 12, siendo esta prueba para muestras menores que 30 .

Por consiguiente, planteamos la hipótesis nula (Ho) e Hipótesis Alternativa $(\mathrm{H} 1)$ de la siguiente manera:

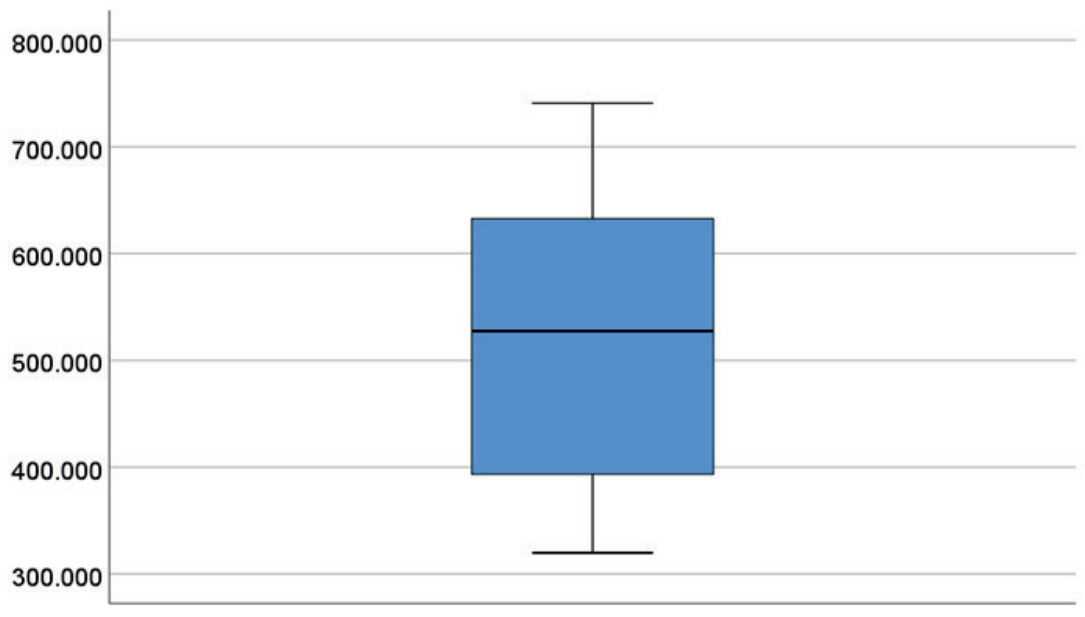

Producto Bruto Interno

Figura 1. Diagrama de cajas del Producto Bruto Interno 2007-2018.

Fuente: Elaboración propia.

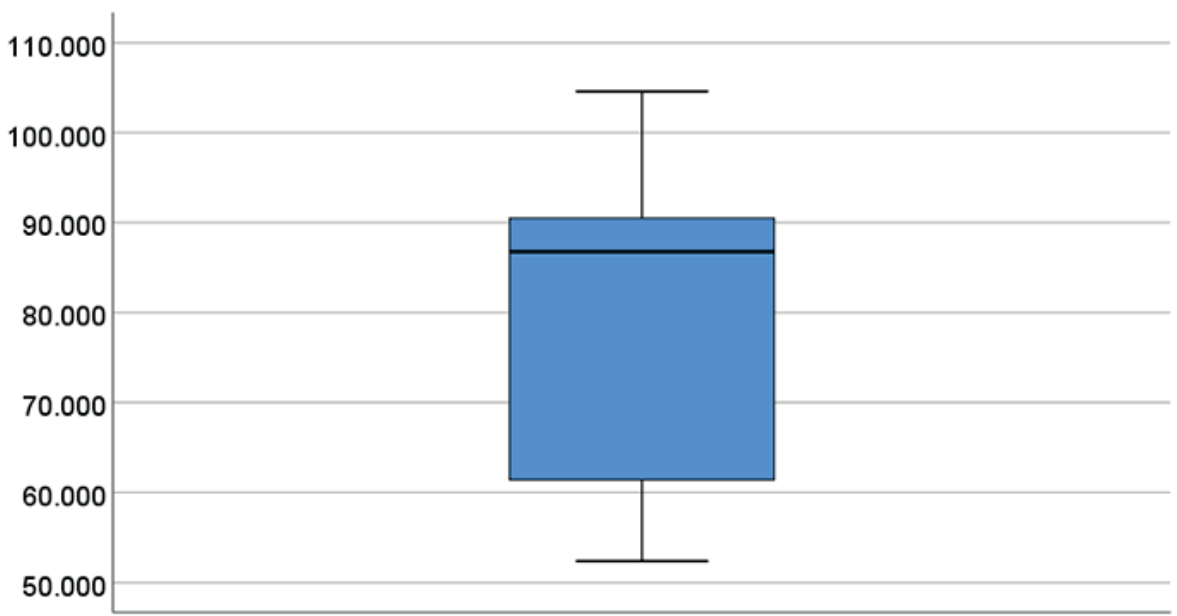

Ingresos Tributarios que recauda el Estado

Figura 2. Diagrama de cajas de los Ingresos Tributarios que recauda el Estado Fuente: Elaboración propia. 
Ho: El Producto Bruto Interno y los Ingresos Tributarios que recauda el Gobierno se distribuyen normalmente.

H1: El Producto Bruto Interno y los Ingresos Tributarios que recauda el Gobierno no se distribuyen normalmente.

En la tabla 2, se muestra que el valor significativo del PBI es 0,787 , este es mayor que 0,05 , entonces no se rechaza la hipótesis nula (Ho), por lo tanto, el PBI tiene distribución normal. El valor significativo de los Ingresos Tributarios que recauda el Gobierno es 0,150, este es mayor a 0,05, entonces no se descarta la hipótesis nula (Ho), por lo tanto, los Ingresos Tributarios que recauda el Gobierno tiene distribución normal.

Debido a la normalidad que presentan el PBI y los Ingresos Tributarios que recauda el Gobierno se procede a realizar un análisis de correlación de Pearson. Antes de hacer la prueba de correlación, se construye un diagrama de dispersión con el fin de analizar visualmente el comportamiento el PBI y los Ingresos Tributarios que recauda el Gobierno.

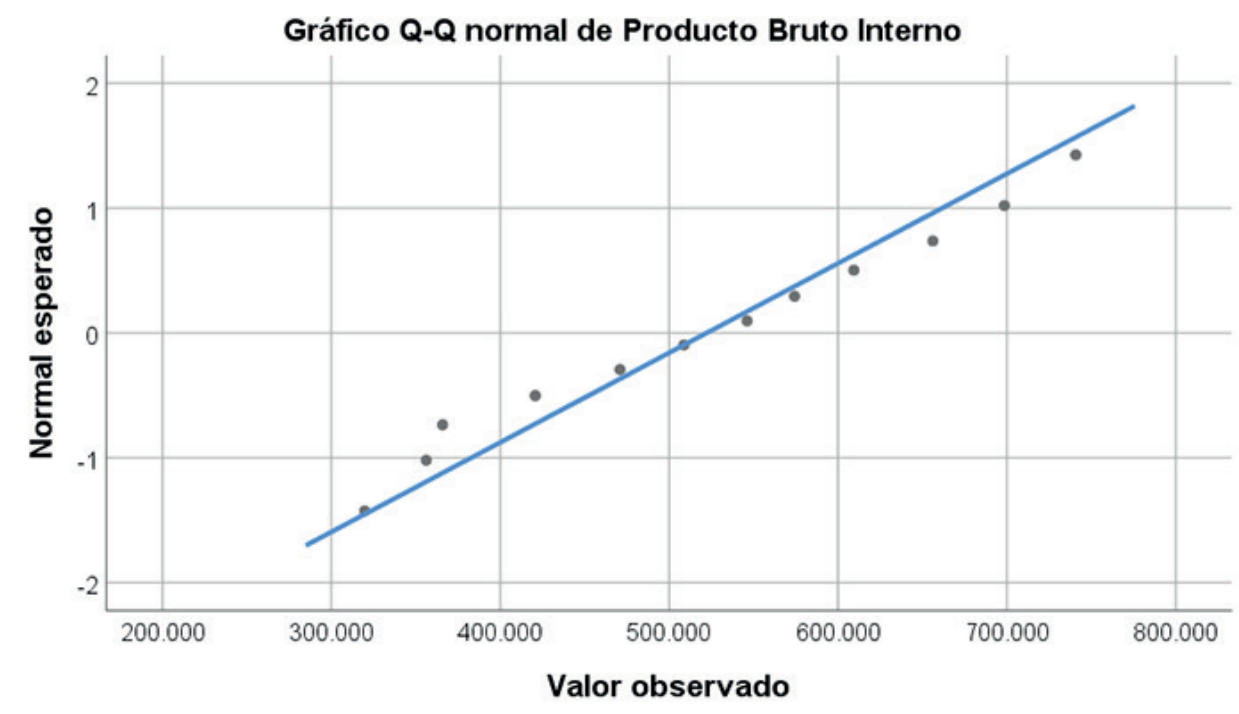

Figura 3. Grafico Q-Q del Producto Bruto Interno.

Fuente: Elaboración propia.

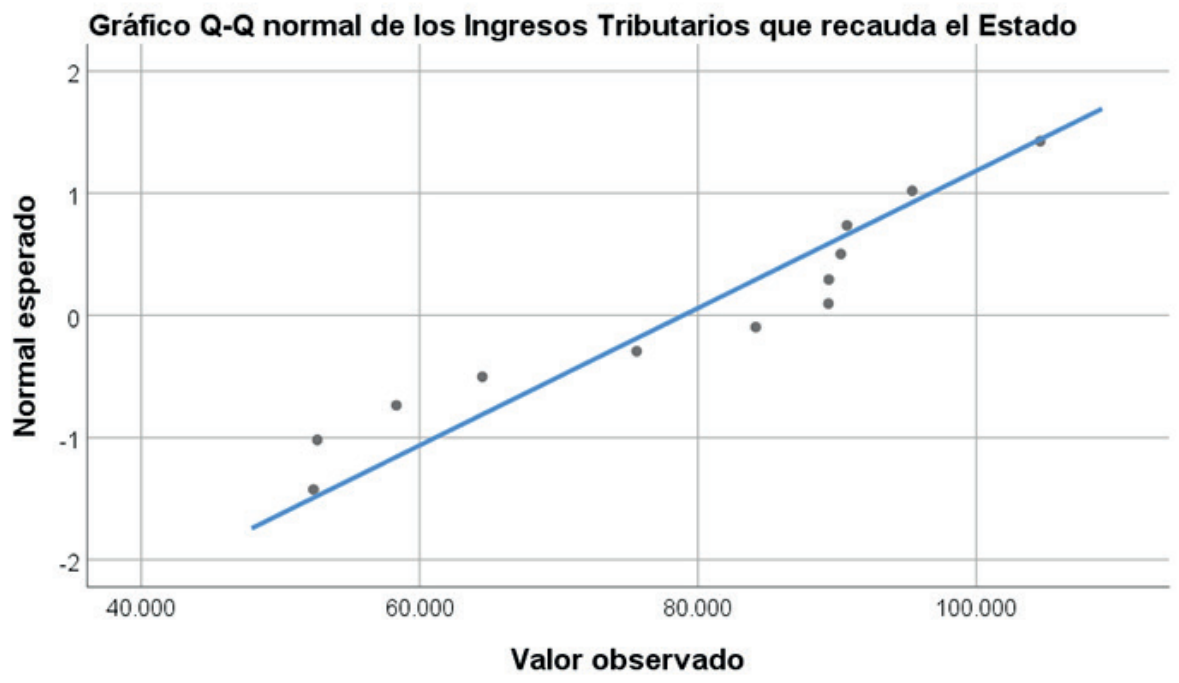

Figura 4. Grafico Q-Q de los Ingresos Tributarios que recauda el Gobierno Central (2007-2018)

Fuente: Elaboración propia. 
En la Figura 5, se observa que los datos se comportan de manera aproximadamente lineal, de forma directa o positiva, es decir cuando aumentan los Ingresos Tributarios que recauda el Gobierno, se incrementa también el PBI, la forma de este diagrama da una idea visual de la alta correlación entre el PBI y los Ingresos Tributarios que recauda el Estado Peruano.

Para probar la hipótesis planteada si existe relación entre PBI y los Ingresos Tributarios que recauda el Estado Peruano, se aplicó el análisis de correlación de Pearson.

Ho: No existe una relación significativa entre el Producto Bruto Interno y los Ingresos Tributarios que recauda el Estado Peruano.

H1: Existe una relación significativa entre el Producto Bruto Interno y los Ingresos Tributarios que recauda el Estado peruano.

En la tabla 3, se muestra que el valor significativo 0,0000055 es menor que 0,05 , entonces rechazamos la hipótesis nula (Ho), en conclusión, existe una relación significativa entre el PBI y los Ingresos Tributarios que recauda el Estado peruano. La dirección de esta relación se corrobora con el coeficiente de correlación de Pearson 0,9401, con este valor se deduce que hay una alta correlación positiva o directa entre el PBI y los Ingresos Tributarios que recauda el Estado peruano. El análisis de estos resultados se explicará en la discusión del estudio.

\section{DISCUSIÓN}

Se ha demostrado estadísticamente que hay una relación significativa entre los Ingresos Tributarios que recauda el Gobierno Central y el PBI. Según el diagrama de dispersión de las variables y el coeficiente de correlación alto de 0,94 , se deduce que la relación entre el PBI e Ingresos Tributarios es positiva o directa, es decir, si aumenta el ingreso tributario, el PBI se incrementa positivamente. Podemos coincidir con lo expuesto por Ordoñez (2014), en la medida que crezca la actividad económica del país, se espera que exista una mayor recaudación tributaria, tanto directa como indirecta. Como resultado del aumento de los Impuestos Tributarios y el aumento del $\mathrm{PBI}$, se origina un incremento de la demanda interna (consumo privado, gasto público e inversión privada)

Tabla 2

Pruebas de normalidad

\begin{tabular}{|c|c|c|c|c|c|c|}
\hline & \multicolumn{3}{|c|}{ Kolmogorov-Smirnova } & \multicolumn{3}{|c|}{ Shapiro-Wilk } \\
\hline & Estadístico & gl & Sig. & Estadístico & gl & Sig. \\
\hline Producto Bruto Interno & 0,119 & 12 & $0,200^{*}$ & 0,960 & 12 & 0,787 \\
\hline Ingresos Tributarios que recauda el Gobierno & 0,221 & 12 & 0,109 & 0,898 & 12 & 0,150 \\
\hline
\end{tabular}

Fuente: Elaboración propia.

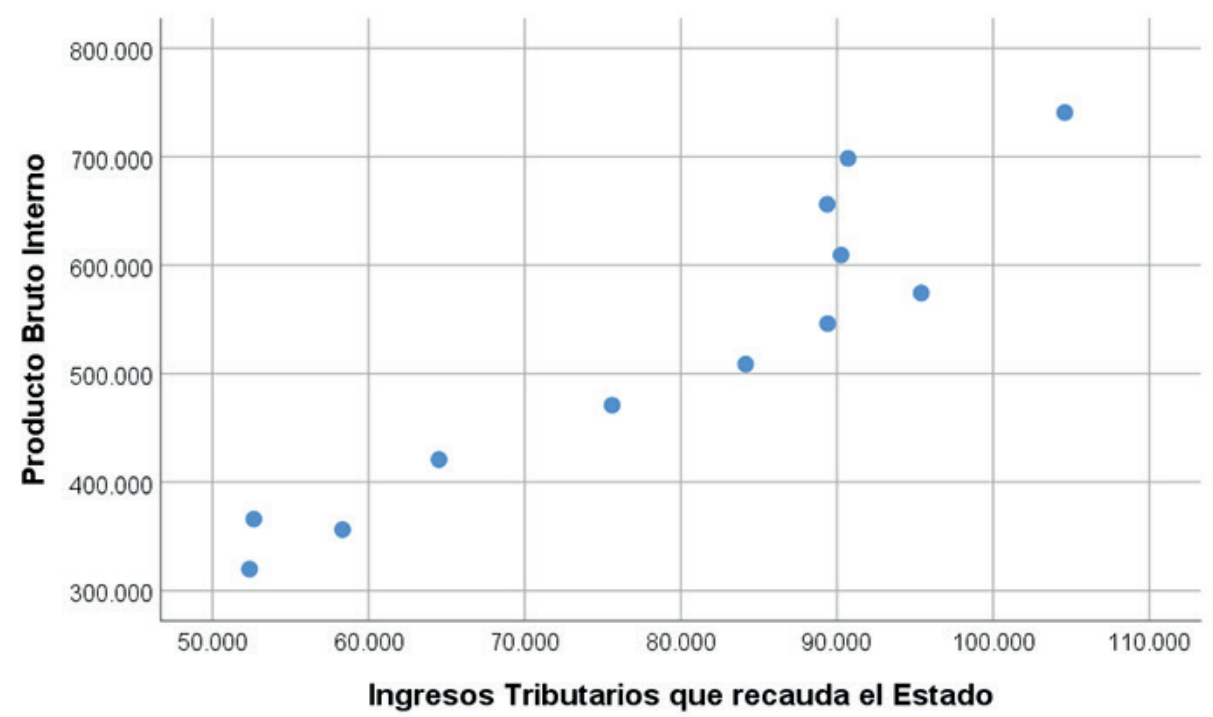

Figura 5. Diagrama de Dispersión del Producto Bruto Interno y los Ingresos Tributarios que recauda el Gobierno Central.

Fuente: Elaboración propia. 
Tabla 3

Correlación de Pearson entre el Producto bruto interno y los Ingresos Tributarios que recauda el Estado.

\begin{tabular}{|c|c|c|c|}
\hline \multicolumn{4}{|c|}{ Correlaciones } \\
\hline & & Producto Bruto Interno & Impuesto Tributario \\
\hline \multirow{3}{*}{ Producto Bruto Interno } & Correlación de Pearson & 1 & $9401^{* *}$ \\
\hline & Sig. (bilateral) & & ,0000055 \\
\hline & $\mathrm{N}$ & 12 & 12 \\
\hline \multirow{3}{*}{ Impuesto Tributario } & Correlación de Pearson & $9401^{* *}$ & 1 \\
\hline & Sig. (bilateral) & ,0000055 & \\
\hline & $\mathrm{N}$ & 12 & 12 \\
\hline
\end{tabular}

**. La correlación es significativa en el nivel 0,01 (bilateral).

Fuente: Elaboración propia.

y externa (exportaciones). De estas actividades, la más importante es el consumo privado dado que representa el $66,35 \%$ de la demanda interna, por lo que su aumento es fundamental para reactivar la economía. La política económica se debe caracterizar por crear un marco que favorezca la inversión exterior y acelerar la integración del país en los mercados mundiales, a fin de que se realice la inversión privada en la minería para que favorezca el incremento de precios de los minerales en el exterior e incrementen las exportaciones, de manera que el país se vea favorecido en la recaudación tributaria. Asimismo, se debe considerar otros elementos para reactivar la economía, como por ejemplo la demanda interna, es decir, se necesita consumir más y para ello es necesario tener más ingresos, por lo tanto, las empresas de todo tamaño deben generar ingresos que permitan otorgan mayores sueldos y salarios a los trabajadores, de esa manera, se podrá gastar más y reactivar el consumo privado y, por ende, la producción. Por ello, es determinante el aumento de la inversión privada, la cual se logra a través de factores internos, externos y políticas de Gobierno, que inciden en el aumento de la recaudación tributaria y PBI.

\section{REFERENCIAS BIBLIOGRÁFICAS}

Banco Central de Reserva del Perú (2019). Glosario de Términos Económicos. Lima. Recuperado de http:// www.bcrp.gob.pe/publicaciones/glosario/p.html
Banco Central de Reserva del Perú (2018). Memoria anual BCRP 2018. Recuperado de http://www.bcrp.gob.pe/ publicaciones/memoria-anual/memoria-2018.html

De Gregorio, J. (2007). Macroeconomía. Teoría y Políticas. Santiago, Chile. Recuperado de http://www.degregorio.cl/pdf/Macroeconomia.pdf

Lahura, E. y Castillo,G. (2018). El efecto de cambios tributarios sobre la actividad económica en Perú: Una Ampliación del enfoque Narrativo. Revista Estudios Económicos, (36), 31-53. Recuperado de http:// www.bcrp.gob.pe/docs/Publicaciones/Revista-Estudios-Economicos/36/ree-36-castillo-lahura.pdf

Moreno, J. (2018). Influencia del PBI y la inflación en el ingreso tributario del Perú, periodo 2003-2017 (tesis para obtener el título profesional de economista con mención en finanzas). Universidad Privada Antenor Orrego, Trujillo, Perú.

Ordoñez, A. (2014) Crecimiento económico e ingresos tributarios del Perú. Logos, 4 (1), 1-8. Recuperado de: http://revistas.uap.edu.pe/ojs/index.php/LOGOS/article/view/302/220

Parodi,C. (2019). ¿Se puede reactivar la economía? Diario Gestión. Recuperado de https://gestion.pe/blog/economiaparatodos/2019/09/se-puede-reactivar-la-economia.html/ 
